\title{
Fault Location in Low-Voltage Distribution Networks based on Reflectometry - A Case Study
}

\author{
J. Ballestín-Fuertes ${ }^{1}$, D. Cervero ${ }^{1}$, H. Bludszuweit ${ }^{1}$, R. Martínez $^{2}$ and Jose Antonio Saez Castro ${ }^{3}$ \\ ${ }^{1}$ CIRCE Foundation \\ Parque Empresarial Dinamiza, Ave. Ranillas 3D, 1st Floor \\ 50018 Zaragoza (Spain) \\ Phone number: +0034 976976 859, e-mail: jballestin@fcirce.es, dcervero@fcirce.es, hbludszuweit@fcirce.es
}

${ }^{2}$ Advanced Electro-Energetic Technologies Group, Department of Electrical Engineering

E.T.S.I.I. University of Cantabria, Av. Los Castros

39005 Santander (Spain)

e-mail: raquel.martinez@unican.es

${ }^{3}$ Viesgo Distribución

Parque Científico Tecnológico, C/ Isabel Torres 25

39011 Santander (Spain)

Phone number: +0034 942246 000, e-mail: jose.antonio.saez@viesgo.com

\begin{abstract}
.
Fault location can help transport and distribution system operators in their effort of minimizing supply interruption times. Nowadays, fault location devices are widely extended in the transport grid. However, the application of these solutions to distribution networks is not a feasible option due to the high cost of this equipment. Therefore, current research is focussed on costeffective fault location techniques which are adapted to electrical distribution networks.

This paper presents results of a case study, conducted with detailed simulation models of two actual low-voltage (LV) distribution grids, using PSCAD software. One is a typical rural grid with long aerial lines, while the other is a typical urban grid with shorter line lengths which are mostly installed underground. The analysis is focussed on fault location based on travelling wave theory and reflectometry methods. The simulations include distributed parameter line models and a signal injector, in order to analyse the singular effects in the waveform which are caused by the special features of the LV network. It is shown that LV networks have some unique features which are not present in medium and highvoltage grids, which makes effective fault location more challenging. Observed issues are discussed and future work is proposed in order to overcome some of them.
\end{abstract}

Key words. Fault location, Power distribution, Low Voltage, PSCAD, Reflectometry

\section{Introduction}

Minimizing supply interruption times is a main goal for distribution system operators (DSO). This objective can be achieved using fault location techniques because fast and precise failure location allows maintenance crews to repair the problem earlier.
According to the literature, reflectometry is the most accurate method for fault location. This technique consists in studying the reflections of electrical signals when they travel through the network. Whenever these travelling waves find abrupt impedance changes, they suffer reflection and refraction, whereby a part of the wave continues travelling forward while a reflected signal moves backwards [1], [2]. Impedance changes can be produced by a transition from one kind of cable to another (i.e. aerial to underground), a line fork or the presence of a load. All these reflections can be measured and create the "line response".

Although advanced tools exist for fault location in the high-voltage (HV) transmission system, on the contrary, this is an unsolved problem in the distribution grid [3]. Several studies have been published focusing on fault location in medium-voltage (MV) networks, but there are very few references related to fault location in low-voltage (LV) grids [4]-[8]. While it has been shown that methods applied to HV systems are applicable for MV, this is not the case for LV networks. The LV grid has a variety of specific characteristics which require alternative approaches. Therefore, further research is needed to obtain a feasible fault location method for low-voltage distribution networks.

One important difference between MV and LV systems is the presence of a shield in MV underground lines in order to conduct unbalance and ground fault currents of the system. LV underground cables do not have this metallic layer. Therefore, a high-frequency signal injected into a LV underground line is not confined within the cable (travelling within the insulator layers between core and 
shield), but between the line core and the neutral core. Due to this feature the propagation characteristics do not only depend on the wire insulator properties but also on the environment surrounding the LV line [9], [10]. Another important difference consists in the end points of the systems according to the travelling waves. MV lines start and end in transformers, which are located in secondary electrical substations, while LV lines start at a transformer, but lead the signals to grounded customer loads, whose reflection characteristic depends on the loading at each time instant.

This paper presents results of a case study conducted on a rural and an urban grid model. From this analysis, several fundamental challenges have been identified when employing reflectometry to locate faults in LV grids. Finally, possible solutions are formulated, whose implementation are the subject of ongoing research conducted by the authors.

\section{Proposed fault location method}

The proposed method [11], [12], [13] consists of a device which periodically injects a high frequency signal into the electrical grid and logs the line response with the aim of creating a reference image of the healthy grid, under prefault conditions. When a fault occurs, the device injects the same signal again. The fault represents a new discontinuity in the grid and produces an additional reflection which was not present in the pre-fault line response. By comparing both images, the reflection in the fault point can be identified, as illustrated in Figure 1.

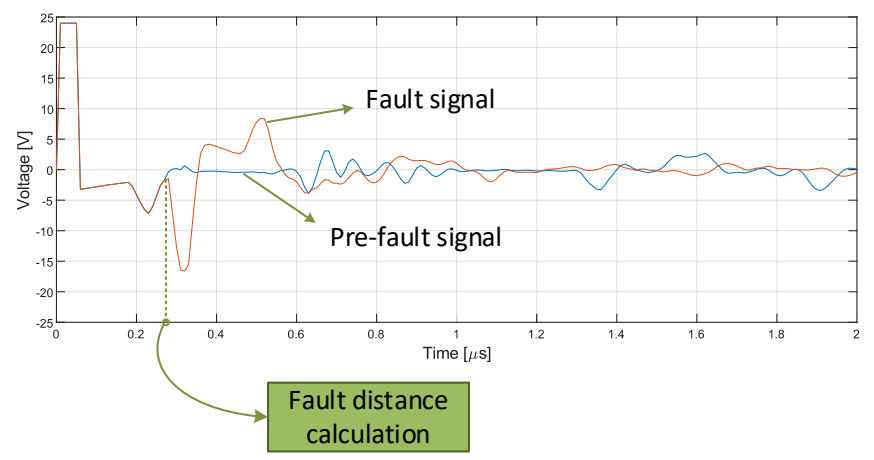

Figure 1: Illustration of the proposed fault location method.

Comparing the fault response with the reference, the time can be measured, when signals start to diverge. This time can be converted into fault distance $(d)$, if signal propagation speed $(v)$ is known, applying expression (1).

$$
d=\frac{v \cdot t}{2}
$$

where $v$ is the propagation speed of the injected signal through the electrical network, $t$ is the time where the fault was located.

Equation (1) contains a division by two since measurement $t$ represents the time interval spent by the injected signal reaching the fault point and return to the device, so twice the time is needed to travel from the device to the fault.

\section{Low voltage grid modelling}

\section{LV electrical distribution grid}

Most low-voltage networks can be grouped according to two typologies: rural and urban grids. Both usually have a radial topology but there are significant differences which require analysing these cases separately. On the one hand, rural grids typically consist of long overhead lines with relatively few loads. On the other hand, urban grids are mostly composed of underground lines with a higher density of consumers than the rural cases, covering shorter distances.

In order to study the application of reflectometry in the LV grid covering most potential cases, one rural and one urban scenario have been considered, implementing detailed models for each case. These two models are based on actual distribution networks whose parameters are taken from real data provided by a Spanish DSO.

In order to achieve certain representativeness, the two studied grids have been chosen according to the following criteria:

- Most of grid parameters should be quantified (i.e. length of sections, loads, cable model).

- The total length of the grid must be long enough in order to determine the range of the fault location method. Furthermore, the total length should be representative considering the kind of network (at least $50^{\text {th }}$ percentile). Figure 1 shows the total length histogram of all analysed networks.

- The rural grid should contain at least $75 \%$ overhead lines (regarding line lengths).

- The urban grid should contain at least $75 \%$ of underground cables (regarding line lengths).

- Chosen grids should have a comparatively high number of reported incidences, in order to increase the probability of finding a fault during the pilot phase.

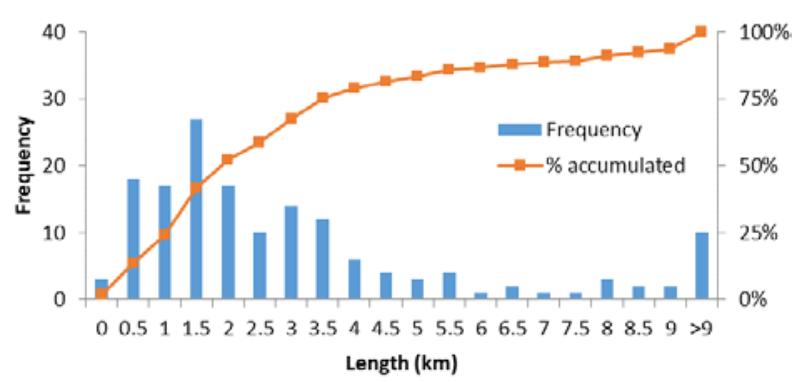

Figure 2. Histogram of total length in rural distribution networks

Firstly, two MV lines, one of them feeding a city and another feeding several small villages, were selected where it was considered feasible to develop a pilot phase in one of their secondary substations. In a second step, all LV networks which are fed from those two MV lines were analysed in order to choose the two most representative LV grids (urban and rural). According to the criteria listed above, two secondary substations were selected to simulate their behaviour. 


\section{Simulation program}

PSCAD has been chosen for this reflectometry study, because it is a simulation environment that allows the creation of networks based on real wires using a transmission line model. The transmission line model allows high-frequency signals to be simulated not only using the classic circuit theory but also as travelling waves. This feature is key to study the behaviour of high-frequency signals and their reflections across the lines.

Figure 2 and Figure 3 show the real LV distribution grid and the accurate simulation model for the rural and the urban grid, respectively.

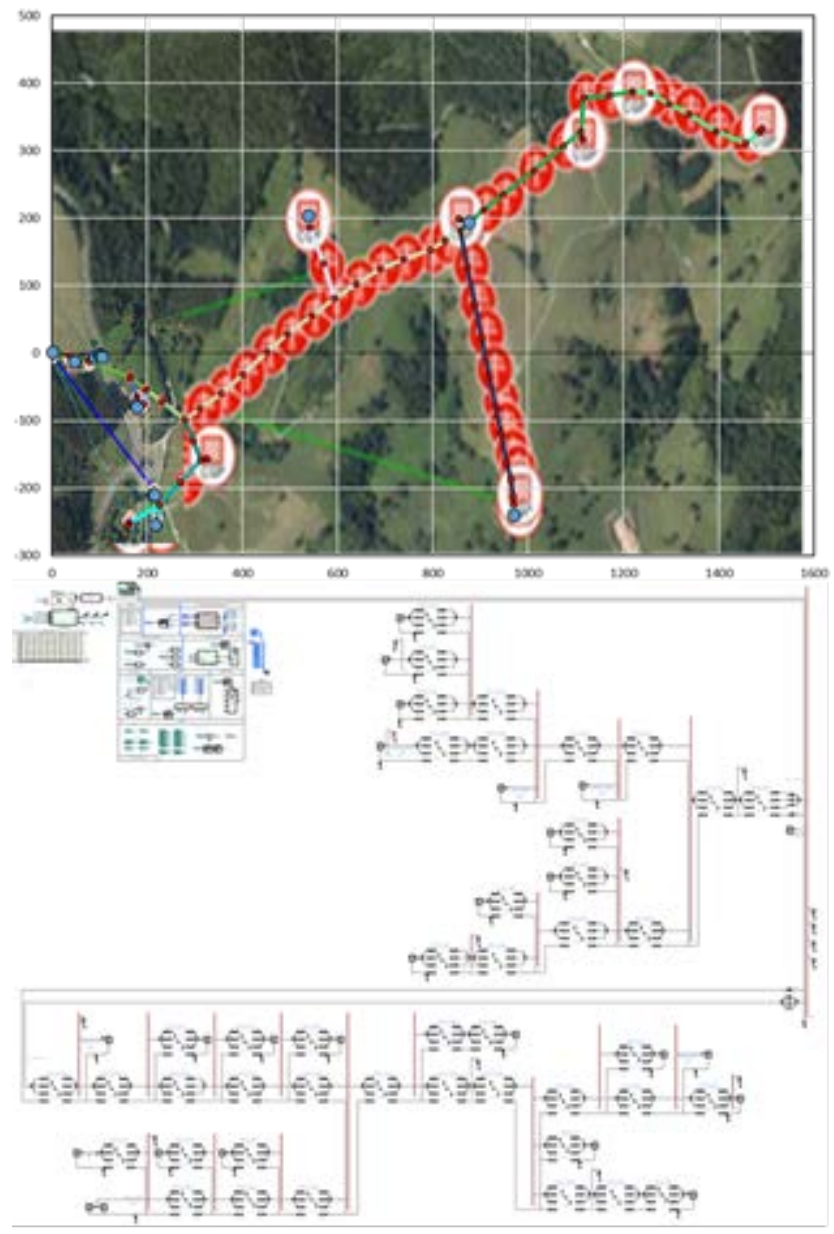

Figure 3: Real grid and simulation model for the rural study case.

\section{Characteristics of the models}

Many factors have been considered in order to implement the models as close to reality as possible. The most important factors are described below:

- Line models have been based on a distributed parameter approach. Nine different underground and aerial wires have been implemented considering all their structural features (mainly dimensions and material properties).

- The load profiles of all consumers supplied by these electrical distribution networks have been analysed in order to implement realistic loads into the models. Overall, more than ninety singlephase and three-phase (balanced and unbalanced) loads have been considered.

- An electrical transformer has been implemented into the model. A programmable short-circuit power at the medium voltage level has been also implemented to study all possible effects to the waveform coming from the LV distribution network equipment.

- A signal injector has been added to the model of both networks. The injector introduces highfrequency signals to the grid through a coupling filter. These signals propagate and reflect through the grid and the reflections are measured at the injector connection point, taking an electric image in form of high-resolution time series.

All components have been distributed through the simulation model according to the real data provided by the DSO.

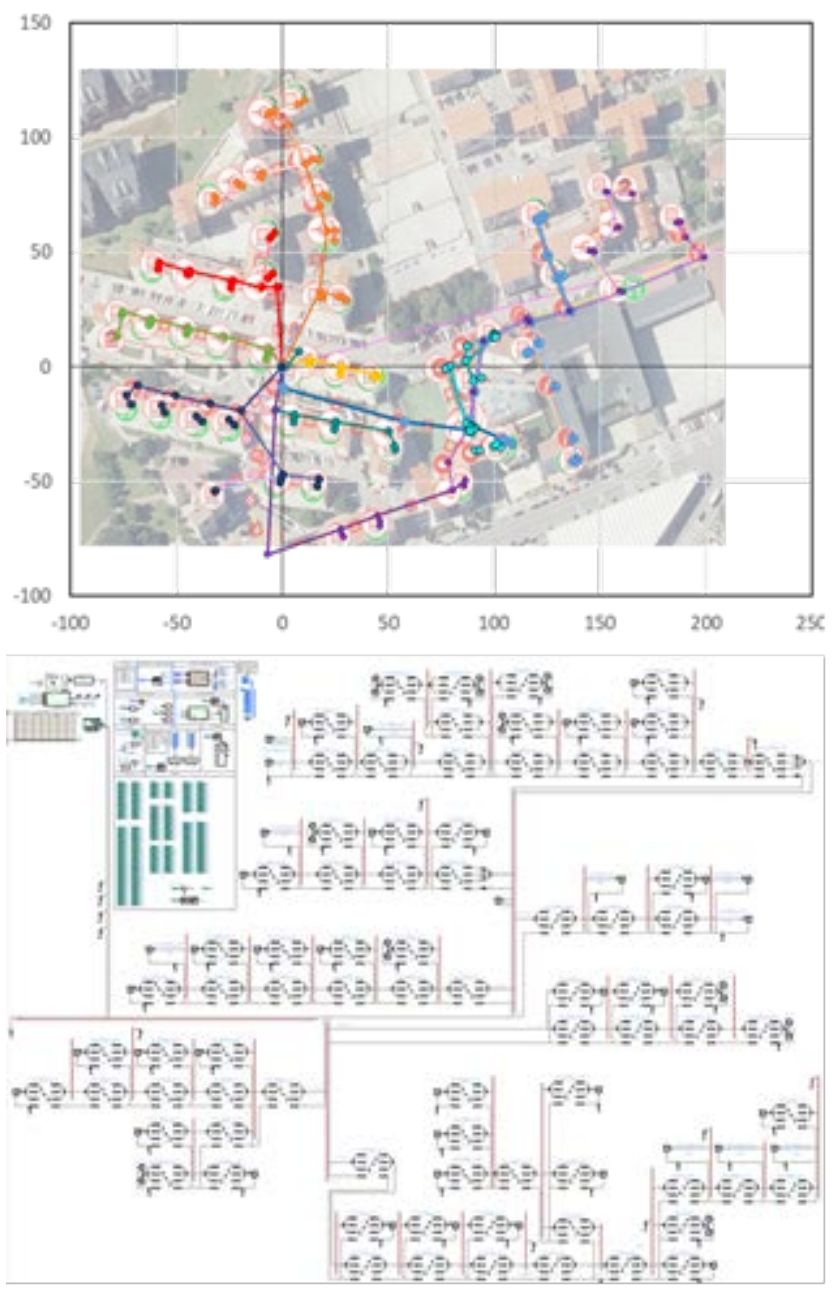

Figure 4: Real grid and simulation model for the urban study case.

An extensive set of simulations has been designed according to several considerations. These parameters are described below:

1) 2 injected signal waveforms: rectangular pulses and sine wavelets of different magnitudes.

2) 4 fault resistances: 0.01, 80, 150 and $1000 \Omega$. 
3) 5 fault types: phase $A$ to ground, phase $B$ to ground, phase $A-B$, phase $B-C$ to ground and phase A-B-C to ground.

4) 10 fault distances from the injector (placed at the head of the line): varying distances between 12 and $1800 \mathrm{~m}$.

PSCAD provides the Multi-run option. This feature allows to carry out several simulations automatically and save the generated data in files that can be processed afterwards. With the Multi-run configuration reported in this paper, 400 simulation cases have been executed for each grid scenario (rural and urban).

\section{Results}

The proposed fault location method relies on the electric waveforms recorded at the secondary electrical substation, at the same point where the injector generates the signal that travels through the lines of the network. Hence, not only the waveforms logged in different simulations were studied but also their dependencies on several parameters which can change either in different moments or in different networks.

\section{Boundary conditions}

A preliminary study of both grid models concluded that the impact on location results the type of the $\mathrm{MV}$ to $\mathrm{LV}$ transformer or the short-circuit power at that common connection point is neglectable, if the injection device (mainly the filter) is designed adequately.

Another study has been devoted to the fact that fluctuation loads in LV grids can cause false positives (fault location at points where there is no fault). As described above, the travelled waveform detects impedance changes throughout the line where it is injected. In this way, a load change between pre-fault and fault instants can be detected wrongly as a fault location.

Figure 5 shows the results of a simulation scenario in which several load values have been changed between the pre-fault and fault instants. The graph show time series of the difference in each phase between the pre-fault and fault signals, measured at the head of the network (where the location device is placed), while the green circle shows the point where the fault should be detected. If there are no load changes between pre-fault and fault injections, the signal difference before this point should be equal to zero. Nevertheless, load changes cause different reflections and, therefore, a high-frequency signal appears before the green circle, similar to waveforms created by an actual fault.

As can be expected, larger fluctuations of customer loads imply a greater amplitude of the noise signal created before the fault instance (green circle). In addition, if the fault impedance is increased, it may become impossible to distinguish load fluctuations from actual faults.

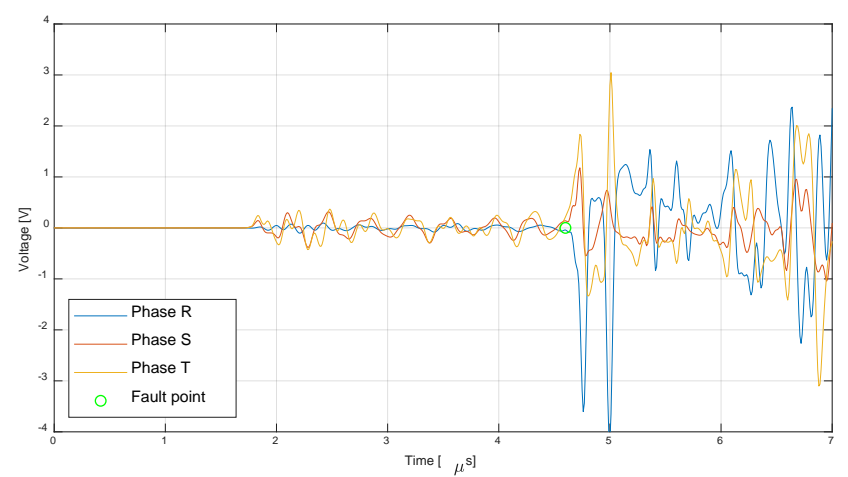

Figure 5: Effect of load fluctuations on the line response.

The location method should face that dependency because load changes occur all the time. When the fault impedance has a low value, the fault location algorithm can distinguish between reflections from load changes and from the fault itself. However, additional efforts are required in order to distinguish reflections from load changes and faults with higher impedances. One proposed alternative consists in a higher frequency of injections to reduce differences between pre-fault and fault scenarios. Another proposed method utilises neural networks to identify fluctuations coming from usual impedance changes (i.e. load fluctuations) and remove them from the location signal.

Once all dependencies have been identified, waveforms obtained according to the previously described set of simulations were studied. In a first approach, waveforms were obtained in an ideal scenario, without any load fluctuations.

\section{Digital signal processing}

A study of the waveform in the frequency domain has been carried out. This study shows that the injected signal creates a series of standing waves inside the line. Every near fault can be located very accurately using these standing waves. On the contrary, accurate results were not obtained considering distant faults.

Figure 6 shows the process followed to determine the fault position by means of the frequency spectrum. The standing waves stablished between the signal injector and the fault point create a repetitive pattern. If the bandwidth between two consecutive "harmonics" $(\Delta f)$ is determined, the fault distance can be calculated according to the following expression:

$$
d=\frac{v}{2 \Delta f}
$$

where $d$ is the distance from the signal injector to the fault and $v$ is the propagation speed. 


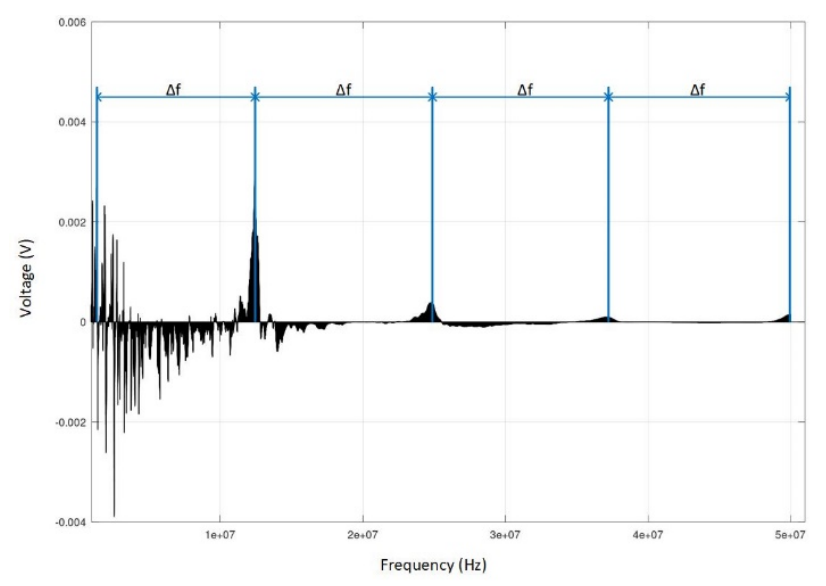

Figure 6: Fast Fourier transform of the simulated signal.

The study of logged waveforms in the time domain gives accurate results even considering far faults from the secondary electrical substation. However, the results are accurate enough taking into account a "time location". But several factors must be considered to calculate the fault distance (the expected result) from the measured fault time. Firstly, the propagation speed of an electromagnetic signal through the lines must be estimated. That velocity factor strongly depends on the electromagnetic permittivity. This relationship is defined by expression (3),

$$
v=\frac{c}{\sqrt{\varepsilon_{r} \mu_{r}}}
$$

where $v$ is the propagation velocity in the medium, $\varepsilon_{r}$ and $\mu_{r}$ the relative permittivity (or dielectric constant) and magnetic permeability respectively, and $c$ the speed of light in vacuum.

Relative magnetic permeability $\mu_{r}$ is approximately equal to one in all materials of interest (usual insulators, air and most of grounds). Therefore, the dielectric constant $\varepsilon_{r}$ can be considered as the only parameter which determines the propagation velocity.

As mentioned before, for unshielded LV cables, the environment surrounding the cable is most relevant for wave propagation. Therefore, for aerial lines $\varepsilon_{r}$ is only slightly above unity, as the predominant medium is air. In this case, the propagation speed is close to the speed of light in the vacuum, with small variations depending on insulator material and core diameters. On the contrary, if cables are buried underground, $\varepsilon_{r}$ varies depending on the ground conditions. Humidity plays a decisive role here, due to the high value of $\varepsilon_{r}=80$ (at $20^{\circ} \mathrm{C}$ ) of water. The impact of humidity also implies that $\varepsilon_{r}$ may change greatly over time. Observations have shown that $\varepsilon_{r}$ can be as much as 14 times higher if the same cable is laid underground instead of aerial.

In addition, signals undergo an attenuation phenomenon along the line. This phenomenon limits the effectiveness of this method up to a certain distance, also known as detection range. This limit is reached when the reflected signal magnitude is similar to the electromagnetic noise. Attenuation and permittivity are strongly related with the propagation constant [14] and, therefore, with the line characteristics defined by the following simplified expression [9], [15],

$$
\gamma=\sqrt{(R+j \omega L)(G+j \omega C)}
$$

where $R, L, G$ and $C$ are the electric parameters and $\gamma$ the propagation constant, whose real term represents the linear losses and the imaginary term represents the phase shift.

In a LV cable, these electric parameters depend not only on the wire properties, but also on the surrounding environment (air or ground).

A laboratory test bench will be set up in order to characterize these parameters and complement simulation results. It will consist of underground cables like those installed in LV distribution networks. They will be arranged according to the most common structures in LV grids (direct-buried and into a flexible plastic conduit). Several tests will be carried out changing the ground properties, for example humidity, in order to determine the influence of different ground properties on wave propagation velocity.

\section{Conclusions}

Fault location is a key objective for DSOs and an unsolved challenge for LV networks. This paper describes a LV simulation scenario where a fault location method based on travelling wave theory and reflectometry techniques has been studied. A Spanish DSO provided detailed information, permitting the creation of two realistic and representative study cases, covering rural and urban distribution networks. These electrical grids have been modelled in PSCAD simulation environment as accurately as possible. Cable types and lengths, load values and the MV connection have been considered and an extensive simulation set has been carried out, considering 400 scenarios for each network.

From simulation results, it can be concluded that both time-domain and frequency-domain analyses are able to detect the fault distance with promising accuracy. Nevertheless, the special features of LV networks introduce several challenges. On the one hand, the electric behaviour of $\mathrm{LV}$ cables is one of the most important handicaps found in this study. Location accuracy and range depend on propagation speed and attenuation, which in turn are strongly related to the surrounding environment. On the other hand, sudden load changes at the consumption points might produce wrong fault location, as the system response is very similar to a fault. A feasible fault location method must overcome this handicap because sudden changes of consumer loads are commonplace (i.e. load switching events). A reduction of time intervals between consecutive signal injections is proposed to minimise this problem.

Several topics for future research are described, in order to solve these issues. A LV cable laboratory is under construction at CIRCE's facilities, to study the electric features of typical LV underground cables and derive most 
relevant parameters, such as ground humidity or configuration underground cable installation. Finally, a demonstration in a real distribution grid is foreseen to validate simulation results and further study possible solutions to the challenges described in this paper.

\section{Acknowledgement}

This research was funded by the "Ministerio de Ciencia, Innovación y Universidades - Agencia Estatal de Investigación" grant number "RTC-2017-6782-3" and the European Union FEDER funds with name "LOcalización de averías, monitorización de estado y Control en redes de bAja TEnsión-LOCATE".
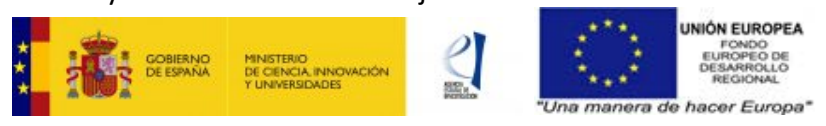

\section{References}

[1] X. X. Aleixandre, Líneas de transmisión. .

[2] R. J. Hamidi and H. Livani, "Traveling-WaveBased Fault-Location Algorithm for Hybrid Multiterminal Circuits," IEEE Transactions on Power Delivery, vol. 32, no. 1. pp. 135-144, 2017.

[3] "Review of fault location methods for distribution power system Review of Fault Location Methods for Distribution Power System,” no. July, 2009.

[4] S. S. Gururajapathy, H. Mokhlis, and H. A. Illias, "Fault location and detection techniques in power distribution systems with distributed generation: A review,” Renew. Sustain. Energy Rev., vol. 74, pp. 949-958, Jul. 2017.

[5] L. Peretto, R. Tinarelli, A. Bauer, and S. Pugliese, "Fault location in underground power networks: A case study,” ISGT 2011. pp. 1-6, 2011.
[6] A. Bahmanyar, S. Jamali, A. Estebsari, and E. Bompard, "A comparison framework for distribution system outage and fault location methods," Electr. Power Syst. Res., vol. 145, pp. 19-34, Apr. 2017.

[7] J. H. Teng, W. H. Huang, and S. W. Luan, "Automatic and Fast Faulted Line-Section Location Method for Distribution Systems Based on Fault Indicators," IEEE Transactions on Power Systems, vol. 29, no. 4. pp. 1653-1662, 2014.

[8] A. Farughian, L. Kumpulainen, and K. Kauhaniemi, "Review of methodologies for earth fault indication and location in compensated and unearthed MV distribution networks," Electr. Power Syst. Res., vol. 154, no. Supplement C, pp. 373-380, 2018.

[9] C. R. Paul, "Multiconductor.” 2007.

[10] R. U. P, "Características eléctricas de la superficie de la Tierra Serie P,” vol. 4, 2017.

[11] M. Abad, S. Borroy, D. López, and M. Garcíagracia, "New Fault Location Method for up-todate and Upcoming Distribution Networks," CIRED 23 rd Int. Conf. Electr. Distrib., no. 1290, 2015.

[12] N. El Halabi, "Localizadores de faltas para redes de distribución eléctrica," Universidad de Zaragoza, 2012.

[13] M. Abad López, “Algoritmos de localización de faltas en redes eléctricas,” Zaragoza, 2016.

[14] F. Costa, M. Borgese, M. Degiorgi, and A. Monorchio, "Electromagnetic characterisation of materials by using transmission/reflection (T/R) devices,” Electron., vol. 6, no. 4, 2017.

[15] M. A. O. Kharraz et al., "Experimental characterization of outdoor low voltage cables for narrowband power line communication,” 2016 Int. Symp. Power Line Commun. its Appl. ISPLC 2016, pp. 138-143, 2016. 